WestVirginiaUniversity
THE RESEARCH REPOSITORY @ Wvu

Faculty Scholarship

1998

\title{
Branch cuts due to finite-temperature quasiparticles
}

H. Arthur Weldon

Follow this and additional works at: https://researchrepository.wvu.edu/faculty_publications

\section{Digital Commons Citation}

Weldon, H. Arthur, "Branch cuts due to finite-temperature quasiparticles" (1998). Faculty Scholarship. 72.

https://researchrepository.wvu.edu/faculty_publications/72

This Article is brought to you for free and open access by The Research Repository @ WVU. It has been accepted for inclusion in Faculty Scholarship by an authorized administrator of The Research Repository@ WVU. For more information, please contact ian.harmon@mail.wvu.edu. 


\title{
Branch cuts due to finite-temperature quasiparticles
}

\author{
H. Arthur Weldon \\ Department of Physics, West Virginia University, Morgantown, West Virginia 26506-6315
}

(Received 12 May 1998; published 1 October 1998)

\begin{abstract}
The branch points of individual thermal self-energy diagrams at $k^{2}=4 m^{2}, k^{2}=9 m^{2}, \ldots$ are shown not to be branch points of the full thermal self-energy. Branch points of the full theory are determined by the complex, temperature-dependent energies of the quasiparticles, defined as the pole location, $k_{0}=\mathcal{E}(\vec{k})$, of the exact retarded propagator. The full retarded self-energy is found to have branch points at $k_{0}=2 \mathcal{E}(\vec{k} / 2)$ and $k_{0}$ $=3 \mathcal{E}(\vec{k} / 3)$ as well as cuts in the space-like region. The discontinuities across the branch cuts are complex. The advanced self-energy is related by reflection to the retarded. [S0556-2821(98)06820-9]
\end{abstract}

PACS number(s): 11.10.Wx, 12.38.Mh, 25.75.-q

\section{INTRODUCTION}

At finite temperature, self-energy functions have more branch cuts and more complicated discontinuities than at zero temperature. The finite-temperature discontinuities have direct physical significance [1-9]. It is possible to compute the discontinuity of a self-energy diagram without having to compute the real part by employing cutting rules that replace certain propagators with Dirac delta functions [10-15]. All the known results about the location of branch cuts and the discontinuities across them apply at each order of perturbation theory. The perturbation theory is defined by choosing free thermal propagators that have poles at the zerotemperature mass $m$. This paper will demonstrate that when perturbation theory is summed, the full self-energy will have branch cuts in different places and with different discontinuities than given in perturbation theory.

\section{A. Example at zero-temperature}

A simple zero-temperature example for a scalar field with interaction $\mathcal{L}_{I}=g \phi^{3} / 6$ will illustrate how higher order corrections can shift the location of branch cuts. Suppose that $m$ is the physical mass, but that one performs perturbative calculations using a free propagator $\Delta(k)=1 /\left(k^{2}-m_{0}^{2}\right)$, where $m_{0}$ is some different mass. For simplicity, $m_{0}$ should be finite and not the bare mass. The one-loop self-energy

$$
\Pi^{(1)}(k)=\frac{i g^{2}}{2} \int \frac{d^{4} p}{(2 \pi)^{4}} \Delta(p) \Delta(p-k)
$$

has a branch cut for $k^{2} \geqslant 4 m_{0}^{2}$. The discontinuity across the branch cut is

$$
\begin{aligned}
\operatorname{Disc} \Pi^{(1)}(k) & =\frac{-i g^{2}}{8 \pi^{2}} \int d^{4} p \delta_{+}\left(p^{2}-m_{0}^{2}\right) \delta_{+}\left[(p-k)^{2}-m_{0}^{2}\right] \\
& =\frac{-i g^{2}}{16 \pi}\left(1-\frac{4 m_{0}^{2}}{k^{2}}\right)^{1 / 2}
\end{aligned}
$$

The indication that $k^{2}=4 m_{0}^{2}$ is not a branch point of the full theory comes from the two-loop contribution. The full propagator is $D^{\prime}(k)=1 /\left[k^{2}-m^{2}-\Pi(k)\right]$ and by definition $\Pi$ contains the necessary counter term to vanish at the true mass $k^{2}=m^{2}$. To do perturbation theory with mass $m_{0}$, the full propagator is written $D^{\prime}(k)=1 /\left[k^{2}-m_{0}^{2}-\widetilde{\Pi}(k)\right]$ where $\widetilde{\Pi}(k)=m^{2}-m_{0}^{2}+\widetilde{\Pi}(k)$. Of course $\tilde{\Pi}$ does not vanish at $k^{2}$ $=m^{2}$ or at $k^{2}=m_{0}^{2}$ and this is the source of the problem. A self-energy insertion on the internal lines of Eq. (1.1) gives the two-loop self-energy term

$$
\Pi^{(2)}(k)=i g^{2} \int \frac{d^{4} p}{(2 \pi)^{4}}[\Delta(p) \tilde{\Pi}(p) \Delta(p)] \Delta(p-k) .
$$

This has a two-particle and a three-particle discontinuity. The quantity in square brackets has a double pole at $p^{2}$ $=m_{0}^{2}$. Using $[\Delta(p)]^{2}=-\partial \Delta(p) / \partial p^{2}$ leads to a two-particle discontinuity

$$
\begin{aligned}
\operatorname{Disc} \Pi^{(2)}(k)= & \frac{i g^{2}}{4 \pi^{2}} \int d^{4} p \delta_{+}^{\prime}\left(p^{2}-m_{0}^{2}\right) \widetilde{\Pi}(p) \\
& \times \delta_{+}\left((p-k)^{2}-m_{0}^{2}\right) .
\end{aligned}
$$

The presence of $\delta^{\prime}\left(p^{2}-m_{0}^{2}\right)$ requires an expansion of the shifted self-energy for $p^{2} \approx m_{0}^{2}$ :

$$
\widetilde{\Pi}\left(p^{2}\right)=\delta m^{2}+\Pi\left(m_{0}^{2}\right)+\left(p^{2}-m_{0}^{2}\right) \Pi^{\prime}\left(m_{0}^{2}\right)+\cdots
$$

where $\delta m^{2}=m^{2}-m_{0}^{2}$. The integrated two-particle discontinuity is

$$
\begin{aligned}
\operatorname{Disc} \Pi^{(2)}(k)= & \frac{i g^{2}}{8 \pi}\left[\frac{\delta m^{2}+\Pi\left(m_{0}^{2}\right)}{k^{2}\left(1-4 m_{0}^{2} / k^{2}\right)^{1 / 2}}\right. \\
& \left.-\Pi^{\prime}\left(m_{0}^{2}\right)\left(1-\frac{4 m_{0}^{2}}{k^{2}}\right)^{1 / 2}\right]
\end{aligned}
$$

The second term changes the coefficient of Eq. (1.2) as required by wave function renormalization. The first terms is more important: It is infinite at $k^{2}=4 m_{0}^{2}$. The infinity is a signal that the correct branch point is not at $k^{2}=4 m_{0}^{2}$. Multiple self-energy insertions on the same skeleton have two effects. First, they modify the coefficient of (1 $\left.-4 m_{0}^{2} / k^{2}\right)^{-1 / 2}$ to be 


$$
\begin{aligned}
\delta m^{2} & +\Pi\left(m_{0}^{2}\right)+\delta m^{2} \Pi^{\prime}\left(m_{0}^{2}\right)+\frac{1}{2}\left(\delta m^{2}\right)^{2} \Pi^{\prime \prime}\left(m_{0}^{2}\right)+\cdots \\
& =\delta m^{2}+\Pi\left(m^{2}\right)=\delta m^{2},
\end{aligned}
$$

where, in the last step, $\Pi\left(\mathrm{m}^{2}\right)=0$ has been used. Thus only $\delta m^{2}$ survives as the coefficient of inverse square root. Multiple self-energy insertions also produce successively higher powers of the inverse square root:

$$
\begin{aligned}
\operatorname{Disc} \Pi(k)= & \frac{-i g^{2}}{16 \pi}\left[\left(1-\frac{4 m_{0}^{2}}{k^{2}}\right)^{1 / 2}-\frac{2 \delta m^{2}}{k^{2}}\left(1-\frac{4 m_{0}^{2}}{k^{2}}\right)^{-1 / 2}\right. \\
& \left.-\frac{1}{2}\left(\frac{2 \delta m^{2}}{k^{2}}\right)^{2}\left(1-\frac{4 m_{0}^{2}}{k^{2}}\right)^{-3 / 2}+\cdots\right] .
\end{aligned}
$$

This is the beginning of a Taylor series. All the corrections diverge at the false threshold $k^{2}=4 m_{0}^{2}$. In the range $4 m_{0}^{2}$ $<k^{2}<4\left(m_{0}^{2}+\left|\delta m^{2}\right|\right)$ each correction is finite, but the Taylor series diverges. Thus perturbation theory fails throughout this region of $k^{2}$. To obtain a convergent series, it is necessary to work in the range $k^{2}>4\left(m_{0}^{2}+\left|\delta m^{2}\right|\right)$. In this region the Taylor series converges and the sum is the full twoparticle discontinuity with branch point shifted to the physical mass $m^{2}=m_{0}^{2}+\delta m^{2}$ :

$$
\operatorname{Disc} \Pi(k)=\frac{-i g^{2}}{16 \pi}\left(1-\frac{4 m^{2}}{k^{2}}\right)^{1 / 2} .
$$

The true two-particle threshold is still a square root branch point at $k^{2}=4 m^{2}$. The breakdown of perturbation theory is entirely due to a propagator $\Delta(k)=1 /\left(k^{2}-m_{0}^{2}\right)$ with the wrong mass $m_{0}$. The breakdown is easily avoided by using $1 /\left(k^{2}-m^{2}\right)$ for the free particle propagator.

\section{B. Non-zero temperature}

In the previous example, individual diagrams of the perturbation series have branch points at the wrong threshold $k^{2}=4 m_{0}^{2}$, although the full theory does not. In finitetemperature field theory, it is customary to perform perturbative calculations using free thermal propagators that have poles at the zero-temperature, physical mass $m$. With this choice, the one-loop self-energy has a branch point at $k^{2}$ $=4 \mathrm{~m}^{2}$. However this is not a true branch point of the full theory. The insertion of the thermal self-energy on an internal propagator produces a two-loop correction analogous to Eq. (1.3) in which there is a double pole at $p^{2}=m^{2}$ because the one-loop self-energy does not vanish there. The double pole produces a discontinuity proportional to (1 $\left.-4 m^{2} / k^{2}\right)^{-1 / 2}$, which diverges at $k^{2}=4 m^{2}$. This claim is easily checked by applying the Kobes-Semenoff cutting rules [10] to compute the discontinuity. Both Le Bellac [14] and Gelis [15] display the two-loop discontinuity as an integral over $d^{4} p$ containing $\delta^{\prime}\left(p^{2}-m^{2}\right)$. This is the same structure as in Eq. (1.4). It is computed explicitly in Appendix A and the result is proportional to $\left(1-4 m^{2} / k^{2}\right)^{-1 / 2}$, which is infinite at the false threshold just as in the $\mathrm{T}=0$ example.

The branch points of the full theory are not obtained by trivially replacing $m^{2}$ by a temperature-dependent effective mass. A proper calculation requires using unperturbed propagators with poles at the same energy at which the thermal self-energy vanishes so that there will be no double poles on internal lines. An energy $\mathcal{E}$ which is a pole of the unperturbed propagator and also a zero of the self-energy is automatically a pole in the full propagator. Poles in the full propagator will occur at energy $k_{0}=\mathcal{E}(\vec{k})$ where $\mathcal{E}$ is is an complicated function of $|\vec{k}|$ that depends on mass, coupling, and temperature. Moreover $\mathcal{E}$ is complex with the imaginary part being the damping rate of the single particle excitation. For definiteness, the real part of $\mathcal{E}$ will be chosen positive and the imaginary part, negative. Thus $\mathcal{E}$ is in the fourth quadrant of the complex energy plane. The pole at $k_{0}=\mathcal{E}(\vec{k})$ is called the quasiparticle pole. This paper will show that there is no branch cut at $k^{2}=4 \mathrm{~m}^{2}$. Instead there is a two-quasiparticle branch point in the full self-energy at the complex, temperature-dependent energy $k_{0}=2 \mathcal{E}(\vec{k} / 2)$. The branch point is the end point of a branch cut in which the two quasiparticles share the energy: $k_{0}=\mathcal{E}\left(\vec{k}_{1}\right)+\mathcal{E}\left(\vec{k}_{2}\right)$ where $\vec{k}$ $=\vec{k}_{1}+\vec{k}_{2}$.

The location of the quasiparticle pole in the full propagator is determined by effects that are higher order in the coupling. Approximating the full propagator by a simpler form that has a pole at the correct position reorders the perturbation series. This is similar to the Braaten-Pisarski resummmation $[16,17]$ of high temperature gauge theories, but differs in several respects. First, the breakdown of perturbation theory near the false thresholds is not an infrared effect. The breakdown occurs even in theories with masses and even if the temperature is small. Second, it is not necessary to retain the $k_{0}$ dependence of the self-energy in the new propagators, only the pole position $\mathcal{E}(\vec{k})$.

A systematic method to organize the reordering of perturbation theory is to employ the integral equation that relates the full self-energy to the exact Minkowski propagator $D_{a b}^{\prime}$ and vertex $\Gamma$ :

$$
\Pi_{a d}(k)=\frac{i g^{2}}{2} \int \frac{d^{4} p}{(2 \pi)^{4}} D_{a b}^{\prime}(p) D_{a c}^{\prime}(p-k) \Gamma_{b c d}(p, p-k) .
$$

Although one does not know the full propagator $D_{a b}^{\prime}$, the natural first approximation is to use a free quasiparticle propagator that has a pole at the correct position. However the Minkowski integral equation is awkward to work with, since it involves propagators with $2^{2}$ components and vertices with $2^{3}$ components. It is simpler to use the imaginarytime formalism because there is only one propagator and one vertex function. In the imaginary-time approach, the full self-energy is related to the full propagator $\mathcal{D}^{\prime}$ and vertex $\Gamma$ by the single integral equation

$$
\begin{aligned}
\Pi(\tau, \vec{k})= & \frac{-g^{2}}{2} \int d \Omega_{12} \int_{0}^{\beta} d \tau^{\prime} d \tau^{\prime \prime} \mathcal{D}^{\prime}\left(\tau^{\prime}, \vec{k}_{1}\right) \mathcal{D}^{\prime}\left(\tau^{\prime \prime}, \vec{k}_{2}\right) \\
& \times \Gamma\left(\tau^{\prime}, \vec{k}_{1} ; \tau^{\prime \prime}, \vec{k}_{2} ; \tau, \vec{k}\right),
\end{aligned}
$$

where $d \Omega_{12}=d^{3} k_{1} d^{3} k_{2} \delta^{3}\left(\vec{k}-\vec{k}_{1}-\vec{k}_{2}\right) /(2 \pi)^{3}$. The existence of a quasiparticle pole at $k_{0}=\mathcal{E}(\vec{k})$ in the Minkowski 
propagator determines the approximation to be used for the Euclidean propagator. By Fourier transforming $\Pi(\tau, \vec{k})$ and then analytically continuing, it is possible to obtain both the retarded and advanced self-energies $\Pi_{R / A}\left(k_{0}, \vec{k}\right)$. This determines everything since each of the four real-time propagators $D_{a b}^{\prime}(k)$ are linear combinations of the retarded and advanced propagators.

The paper is organized as follows. Section II discusses the exact retarded thermal propagator $D_{R}^{\prime}(k)$ and separates the quasiparticle pole from the self-energy effects. Section III introduces the quasiparticle approximation to the propagator in both Minkowski and Euclidean space-time. Section IV computes the one-loop self-energy with quasiparticle propagators. The results are displayed in Eqs. (4.5) to (4.8). The calculations are performed in the imaginary time formalism and then analytically continued to obtain $\Pi_{R / A}$. As a check, Appendix D performs the one-loop calculation entirely in the real-time formalism. The calculation is more difficult, but produces exactly the same results. Section V computes the two self-energy diagrams that contribute at two-loop order. The diagram in which there is a first-order self-energy insertion on an internal line is the direct analogue of Eq. (1.3). Because the quasiparticle self-energy vanishes at $k_{0}=\mathcal{E}$, this diagram does not shift the location of the two quasiparticle branch point. The effect of this contribution is a only a change in the coefficient of the two-quasiparticle cut. Both two-loop diagrams have branch cuts for three-quasiparticle processes and these are computed. Section VI contains the conclusions and the general relation between the real-time $D_{a b}$ and $\Pi_{a b}$ and the retarded or advanced quantities.

\section{EXACT PROPAGATORS}

\section{A. Minkowski space}

The quasiparticle poles occur in the Minkowski-space propagator and it is necessary to begin there and then convert to Euclidean propagators. The exact propagator in Minkowski-space has a $2 \times 2$ matrix structure. All four components are linear combinations of the exact retarded and advanced propagators $D_{R}^{\prime}(k)$ and $D_{A}^{\prime}(k)$ as displayed in Eq. (6.2). Since $D_{A}^{\prime}(k)=D_{R}^{\prime}(-k)$, it suffices to investigate $D_{R}^{\prime}(k)$. The retarded propagator is analytic in the upper-half of the complex $k_{0}$ plane and satisfies the condition

$$
D_{R}^{\prime}\left(k_{0}, \vec{k}\right)=\left[D_{R}^{\prime}\left(-k_{0}^{*}, \vec{k}\right)\right]^{*} .
$$

At zero temperature, the exact propagator has poles at $k_{0}$ $= \pm\left(m^{2}+\vec{k}^{2}\right)^{1 / 2}$. At non-zero temperature, the location of these poles is temperature-dependent and complex. For definiteness, let the pole in the exact retarded propagator that occurs in the fourth quadrant be at $k_{0}=\mathcal{E}$ where

$$
\mathcal{E}(\vec{k})=E(\vec{k})-i \Gamma(\vec{k}) / 2 \quad(E>0 ; \Gamma>0) .
$$

Both $E$ and $\Gamma$ are complicated functions of momentum, temperature, and coupling. The complex energy $\mathcal{E}$ will be called the quasiparticle energy. Because of Eq. (2.1), the retarded propagator must also have a pole in the third quadrant at $k_{0}=-\mathcal{E}^{*}$. Also because of Eq. (2.1), the residues of these two poles are related:

$$
\begin{gathered}
\lim _{k_{0} \rightarrow \mathcal{E}}\left(k_{0}-\mathcal{E}\right) D_{R}^{\prime}(k)=Z / 2 E \\
\lim _{k_{0} \rightarrow-\mathcal{E}^{*}}\left(k_{0}+\mathcal{E}^{*}\right) D_{R}^{\prime}(k)=-Z^{*} / 2 E .
\end{gathered}
$$

Here $Z$ plays the role of the wave-function renormalization constant. The retarded propagator is directly related to the retarded self-energy

$$
D_{R}^{\prime}(k)=\left[k^{2}-m^{2}-\Pi_{R}(k)\right]^{-1} .
$$

Because the full retarded propagator does not have poles at $k^{2}=m^{2}$, the proper self-energy $\Pi_{R}(k)$ does not vanish at $k^{2}=m^{2}$. Therefore the usual Dyson-Schwinger expansion

$$
\frac{1}{k^{2}-m^{2}}+\frac{\Pi_{R}(k)}{\left(k^{2}-m^{2}\right)^{2}}+\frac{\Pi_{R}^{2}(k)}{\left(k^{2}-m^{2}\right)^{3}}+\cdots
$$

is not useful. The first term has a simple pole at $k^{2}=m^{2}$, the second term has a double pole, the third term has a triple pole,... . Performing perturbation theory around $k^{2}=m^{2}$ is quite misleading. It is much better to write the full retarded propagator as

$$
D_{R}^{\prime}(k)=\left[\left(k_{0}-\mathcal{E}\right)\left(k_{0}+\mathcal{E}^{*}\right)-\Pi_{R q p}(k)\right]^{-1},
$$

where the retarded quasiparticle self-energy is defined by

$$
\Pi_{R q p}(k)=\Pi_{R}(k)+\vec{k}^{2}+m^{2}-|\mathcal{E}|^{2}+i \Gamma k_{0} .
$$

By construction, $\Pi_{R q p}(k)$ vanishes at $k_{0}=\mathcal{E}$ and also at $k_{0}$ $=-\mathcal{E}^{*}$ :

$$
\Pi_{R q p}(\mathcal{E})=0 \quad \Pi_{R q p}\left(-\mathcal{E}^{*}\right)=0 .
$$

The natural expansion around the quasiparticle poles is

$$
\frac{1}{\left(k_{0}-\mathcal{E}\right)\left(k_{0}+\mathcal{E}^{*}\right)}+\frac{\Pi_{R q p}(k)}{\left(k_{0}-\mathcal{E}\right)^{2}\left(k_{0}+\mathcal{E}^{*}\right)^{2}}+\cdots \text {. }
$$

The second term has only simple poles at $k_{0}=\mathcal{E}$ and at $k_{0}$ $=-\mathcal{E}^{*}$. It is convenient to define the derivative of the selfenergy at these positions in terms of a complex constant $B$ :

$$
\frac{d \Pi_{R q p}\left(k_{0}\right)}{d k_{0}}=\left\{\begin{array}{l}
2 E B, \quad k_{0}=\mathcal{E}, \\
-2 E B^{*}, \quad k_{0}=-\mathcal{E}^{*} .
\end{array}\right.
$$

This constant $B$ is related to the wave-function renormalization constant in Eq. (2.3) by

$$
Z=1+B+B^{2}+\cdots=1 /(1-B) .
$$

It will be helpful to have similar results for the advanced propagator. From the definition

$$
D_{A}^{\prime}(k)=D_{R}^{\prime}(-k),
$$


the advanced propagator is analytic in the lower-half of the complex $k_{0}$ plane. It must have poles in upper-half plane at $k_{0}=\mathcal{E}^{*}$ and $k_{0}=-\mathcal{E}$. To emphasize these poles, it is convenient to write the advanced propagator as

$$
D_{A}^{\prime}(k)=\left[\left(k_{0}+\mathcal{E}\right)\left(k_{0}-\mathcal{E}^{*}\right)-\Pi_{A q p}(k)\right]^{-1},
$$

where the advanced quasiparticle self-energy is defined to be

$$
\Pi_{A q p}(k)=\Pi_{A}(k)+\vec{k}^{2}+m^{2}-|\mathcal{E}|^{2}-i \Gamma k_{0} .
$$

\section{B. Euclidean space}

The finite-temperature Euclidean propagator is defined at discrete, imaginary frequencies

$$
\omega_{n}=i 2 \pi n T
$$

where $n$ is any integer. The full Euclidean propagator is

$$
\mathcal{D}^{\prime}\left(i \omega_{n}, \vec{k}\right)= \begin{cases}-D_{R}^{\prime}\left(i \omega_{n}, \vec{k}\right) & \text { if } n \geqslant 0 \\ -D_{A}^{\prime}\left(i \omega_{n}, \vec{k}\right) & \text { if } n \leqslant 0\end{cases}
$$

with the overall minus sign chosen for later convenience. Relation (2.12) for $k_{0}$ imaginary implies that $D_{R}^{\prime}(i 2 \pi|n| T, \vec{k})=D_{A}^{\prime}(-i 2 \pi|n| T, \vec{k})$. It follows that $\mathcal{D}^{\prime}\left(i \omega_{n}, \vec{k}\right)$ is an even function of $n$. The Euclidean propagator may be expressed in terms of the self-energy as

$$
\mathcal{D}^{\prime}\left(i \omega_{n}, \vec{k}\right)=\left[\left(\omega_{n}\right)^{2}+\vec{k}^{2}+m^{2}+\Pi\left(i \omega_{n}, \vec{k}\right)\right]^{-1}
$$

where the Euclidean self-energy is

$$
\Pi\left(i \omega_{n}, \vec{k}\right)= \begin{cases}\Pi_{R}\left(i \omega_{n}, \vec{k}\right) & \text { if } n \geqslant 0, \\ \Pi_{A}\left(i \omega_{n}, \vec{k}\right) & \text { if } n \leqslant 0\end{cases}
$$

The relations $\Pi_{R}\left(k_{0}, \vec{k}\right)=\left[\Pi_{R}\left(-k_{0}^{*}, \vec{k}\right)\right]^{*}$ and $\Pi_{A}\left(k_{0}, \vec{k}\right)$ $=\left[\Pi_{A}\left(-k_{0}^{*}, \vec{k}\right)\right]^{*}$ guarantee that Eq. (2.16) is real. To emphasize the quasiparticle aspect, the propagator may be written

$\mathcal{D}^{\prime}\left(i \omega_{n}, \vec{k}\right)=\left[-\left(i\left|\omega_{n}\right|-\mathcal{E}\right)\left(i\left|\omega_{n}\right|+\mathcal{E}^{*}\right)+\Pi_{q p}\left(i \omega_{n}, \vec{k}\right)\right]^{-1}$

where the quasiparticle self-energy is

$\Pi_{q p}\left(i \omega_{n}, \vec{k}\right)=\Pi\left(i \omega_{n}, \vec{k}\right)+\vec{k}^{2}+m^{2}-|\mathcal{E}|^{2}-\Gamma\left|\omega_{n}\right|$

The presence of $|n|$ rather that $n$ in these results is very important, but will cause complications later.

\section{QUASIPARTICLE PROPAGATOR}

The natural approximation to the full Minkowski-space propagators is to retain the quasiparticle poles. Thus approximate (2.6) and (2.13) by

$$
\begin{aligned}
& D_{R}(k)=\frac{1}{\left(k_{0}-\mathcal{E}\right)\left(k_{0}+\mathcal{E}^{*}\right)} \\
& D_{A}(k)=\frac{1}{\left(k_{0}+\mathcal{E}\right)\left(k_{0}-\mathcal{E}^{*}\right)} .
\end{aligned}
$$

The corresponding Euclidean propagator for free quasiparticles follows from Eq. (2.17):

$$
\begin{aligned}
\mathcal{D}\left(i \omega_{n}, \vec{k}\right) & =\frac{-1}{\left(i\left|\omega_{n}\right|-\mathcal{E}\right)\left(i\left|\omega_{n}\right|+\mathcal{E}^{*}\right)} \\
& =\frac{1}{\omega_{n}^{2}+\Gamma\left|\omega_{n}\right|+|\mathcal{E}|^{2}} .
\end{aligned}
$$

The transform to Euclidean time requires the Fourier summation

$$
\mathcal{D}(\tau, \vec{k})=T \sum_{n=-\infty}^{\infty} e^{-i \omega_{n} \tau} \mathcal{D}\left(i \omega_{n}, \vec{k}\right)
$$

for $-\beta \leqslant \tau \leqslant \beta$. Since Eq. (3.2) is an even function of the integer $n$, Eq. (3.3) is automatically an even function of $\tau$. To perform the summation, it is convenient to write Eq. (3.2) without the absolute value bars on $n$ as

$$
\frac{-1}{\left(i \omega_{n}-\mathcal{E}\right)\left(i \omega_{n}+\mathcal{E}^{*}\right)}-\theta(-n) \frac{2 i \omega_{n}\left(\mathcal{E}^{*}-\mathcal{E}\right)}{\left(\omega_{n}^{2}+\mathcal{E}^{2}\right)\left(\omega_{n}^{2}+\mathcal{E}^{* 2}\right)}
$$

Using this gives for the Fourier sum

$$
\begin{aligned}
\mathcal{D}(\tau, \vec{k})= & \frac{1}{2 E}\left\{[1+n(\mathcal{E})] e^{-\mathcal{E}|\tau|}+n\left(\mathcal{E}^{*}\right) e^{\mathcal{E}^{*}|\tau|}\right\} \\
& -T \sum_{n=1}^{\infty} e^{i \omega_{n}|\tau|} \frac{2 \Gamma \omega_{n}}{\left(\omega_{n}^{2}+\mathcal{E}^{2}\right)\left(\omega_{n}^{2}+\mathcal{E}^{* 2}\right)},
\end{aligned}
$$

where the Bose-Einstein function is

$$
n(\mathcal{E})=1 /[\exp (\beta \mathcal{E})-1]
$$

This is the form of the quasiparticle propagator that will be used in the subsequent self-energy calculations. (Le Bellac and Mabilat [18] also use this form for the regularized form of the free thermal propagator, in which case $\mathcal{E}$ is replaced by the free particle energy and $\Gamma$ becomes infinitesimal.) All the $\tau$ dependence in Eq. (3.4) is of the form $\exp (-\Lambda|\tau|)$ where $\Lambda$ is a member of the set below

$$
\Lambda \in\left\{\mathcal{E},-\mathcal{E}^{*},-i \omega_{1},-i \omega_{2},-i \omega_{3}, \ldots\right\} \operatorname{Im} \Lambda<0
$$

Each $\Lambda$ has a negative imaginary part. The propagator will be written compactly as

$$
\mathcal{D}(\tau)=\sum_{\Lambda} f(\Lambda) e^{-\Lambda|\tau|}
$$

in which the coefficient functions are 


$$
\begin{aligned}
f(\mathcal{E}) & =[1+n(\mathcal{E})] / 2 E \\
f\left(-\mathcal{E}^{*}\right) & =n\left(\mathcal{E}^{*}\right) / 2 E \\
f\left(-i \omega_{\ell}\right) & =-2 T \Gamma \omega_{\ell} /\left(\omega_{\ell}^{2}+\mathcal{E}^{2}\right)\left(\omega_{\ell}^{2}+\mathcal{E}^{* 2}\right) .
\end{aligned}
$$

Although Eq. (3.4) will be used throughout, the infinite sum conceals several properties that are important to note. First, the time dependence $\exp (-\Lambda \mid \tau)$ with $\operatorname{Im} \Lambda<0$ will lead to a Euclidean self-energy that can be easily extended to the retarded self-energy in Minkowski space. However the starting point $\mathcal{D}\left(i \omega_{n}, \vec{k}\right)$ in Eq. (3.2) favors neither the retarded nor the advanced forms. Although it is not apparent, Eq. (3.4) is actually real:

$$
\mathcal{D}(\tau, \vec{k})^{*}=\mathcal{D}(\tau, \vec{k}) .
$$

This allows the time dependence to also be written $\exp \left(-\Lambda^{*}|\tau|\right)$ if continuation to the advanced form of the Minkowski self-energy is desired. Although Eq. (3.8) is not obvious, it must be true, since $\mathcal{D}\left(i \omega_{n}, \vec{k}\right)$ is real and an even function of $n$. Appendix B proves Eq. (3.8) explicitly. Second, since $\exp \left(i \omega_{n} \beta\right)=1$, the quasiparticle propagator (3.3) satisfies the KMS condition

$$
\mathcal{D}(\beta-\tau, \vec{k})=\mathcal{D}(\tau, \vec{k}) .
$$

Without the infinite sum in Eq. (3.4), the KMS property would not hold. Appendix B proves Eq. (3.9) explicitly. Third, another way to obtain Eq. (3.4) is to begin with the time-ordered propagator in Minkowski space, which is given by the following linear combination of the retarded and advanced propagators:

$$
D_{11}(k)=\frac{\left[1+n\left(k_{0}\right)\right]}{\left(k_{0}-\mathcal{E}\right)\left(k_{0}+\mathcal{E}^{*}\right)}-\frac{n\left(k_{0}\right)}{\left(k_{0}+\mathcal{E}\right)\left(k_{0}-\mathcal{E}^{*}\right)} .
$$

The Fourier transform, $D_{11}(t, \vec{k})$, for real positive time $t$ is determined by all the poles in the lower-half of the complex $k_{0}$ plane. These poles are at $k_{0}=\mathcal{E}, k_{0}=-\mathcal{E}^{*}$ and at $k_{0}$ $=-i \omega_{n}$ for $n>0$. The propagator in Euclidean time results from continuing from positive, real $t$ to negative, imaginary time $-i \tau$. The Euclidean propagator is $\mathcal{D}(\tau, \vec{k})=i D_{11}$ $(-i \tau, \vec{k})$ and gives precisely Eq. (3.4).

\section{ONE-LOOP SELF-ENERGY}

It is always easy to perform loop corrections by integrating over Euclidean time and then Fourier transforming $[1,19]$. That method will be employed here. The first approximation to the integral equation (1.6) for the full selfenergy is to use the quasiparticle propagator (3.4) and the bare vertex without corrections. This approximation treats the energy $\mathcal{E}$ exactly even though it is a function of the coupling $g$. The one-loop correction shown in Fig. 1 is

$$
\Pi^{I}(\tau, \vec{k})=\frac{-g^{2}}{2} \int d \Omega_{12} \mathcal{D}\left(\tau, \vec{k}_{1}\right) \mathcal{D}\left(\tau, \vec{k}_{2}\right)
$$

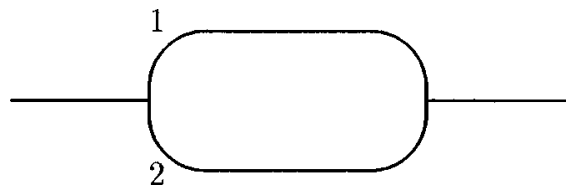

FIG. 1. One-loop self-energy.

This may be expressed concisely using the notation (3.6) for the propagators:

$$
\Pi^{I}(\tau, \vec{k})=\frac{-g^{2}}{2} \int d \Omega_{12} \sum_{\Lambda_{1}, \Lambda_{2}} f\left(\Lambda_{1}\right) f\left(\Lambda_{2}\right) e^{-\left(\Lambda_{1}+\Lambda_{2}\right)|\tau|} .
$$

The transform from $\tau$ to discrete frequency $\omega_{n}$ is

$$
\begin{aligned}
\Pi^{I}\left(i \omega_{n}, \vec{k}\right) & =\int_{0}^{\beta} d \tau e^{i \omega_{n} \tau} \Pi^{I}(\tau, \vec{k}) \\
& =\frac{g^{2}}{2} \int d \Omega_{12} \sum_{\Lambda_{1}, \Lambda_{2}} f\left(\Lambda_{1}\right) f\left(\Lambda_{2}\right) \frac{1-e^{-\left(\Lambda_{1}+\Lambda_{2}\right) \beta}}{i \omega_{n}-\Lambda_{1}-\Lambda_{2}} .
\end{aligned}
$$

This can be extended from $i \omega_{n}$ for $n>0$ to complex $k_{0}$ with $\operatorname{Im} k_{0}>0$. It is analytic for $\operatorname{Im} k_{0}>0$ because $\Lambda_{1}$ and $\Lambda_{2}$ have negative imaginary parts. The extension therefore gives the retarded self-energy:

$$
\Pi_{R}^{I}\left(k_{0}, \vec{k}\right)=\frac{g^{2}}{2} \int d \Omega_{12} \sum_{\Lambda} f\left(\Lambda_{1}\right) f\left(\Lambda_{2}\right) \frac{1-e^{-\left(\Lambda_{1}+\Lambda_{2}\right) \beta}}{k_{0}-\Lambda_{1}-\Lambda_{2}} .
$$

Although this is analytic for $k_{0}$ in the upper-half of the complex plane, when $k_{0}$ is continued into the lower half-plane, the singularities at $k_{0}=\Lambda_{1}+\Lambda_{2}$ produce branch cuts in the self-energy.

Physical Cuts: It is useful to write out the various cases for the different $\Lambda_{i}$. First, if $\Lambda_{1}=\mathcal{E}_{1}$ and $\Lambda_{2}=\mathcal{E}_{2}$, the contribution to the self-energy is

$$
\frac{g^{2}}{2} \int \frac{d \Omega_{12}}{2 E_{1} 2 E_{2}} \frac{\left[1+n\left(\mathcal{E}_{1}\right)\right]\left[1+n\left(\mathcal{E}_{2}\right)\right]-n\left(\mathcal{E}_{1}\right) n\left(\mathcal{E}_{2}\right)}{k_{0}-\mathcal{E}_{1}-\mathcal{E}_{2}}
$$

The discontinuity across the cut is complex. The statistical factors provide for the Bose-Einstein enhanced emission of two quasiparticles minus the absorption of two quasiparticles. The second contribution is for $\Lambda_{1}=\mathcal{E}_{1}$ and $\Lambda_{2}$ $=-\mathcal{E}_{2}^{*}$ :

$$
\frac{g^{2}}{2} \int \frac{d \Omega_{12}}{2 E_{1} 2 E_{2}} \frac{\left[1+n\left(\mathcal{E}_{1}\right)\right] n\left(\mathcal{E}_{2}^{*}\right)-n\left(\mathcal{E}_{1}\right)\left[1+n\left(\mathcal{E}_{2}^{*}\right)\right]}{k_{0}-\mathcal{E}_{1}+\mathcal{E}_{2}^{*}}
$$

The statistical factors account for a direct process in which quasiparticle 1 is emitted and quasiparticle 2 is absorbed minus the inverse process. If $\Lambda_{1}=-\mathcal{E}_{1}^{*}$ and $\Lambda_{2}=\mathcal{E}_{2}$, the result is 


$$
\frac{g^{2}}{2} \int \frac{d \Omega_{12}}{2 E_{1} 2 E_{2}} \frac{n\left(\mathcal{E}_{1}^{*}\right)\left[1+n\left(\mathcal{E}_{2}\right)\right]-\left[1+n\left(\mathcal{E}_{1}^{*}\right)\right] n\left(\mathcal{E}_{2}\right)}{k_{0}+\mathcal{E}_{1}^{*}-\mathcal{E}_{2}} .
$$

If $\Lambda_{1}=-\mathcal{E}_{1}^{*}$ and $\Lambda_{2}=-\mathcal{E}_{2}^{*}$, the self-energy is

$$
\frac{g^{2}}{2} \int \frac{d \Omega_{12}}{2 E_{1} 2 E_{2}} \frac{n\left(\mathcal{E}_{1}^{*}\right) n\left(\mathcal{E}_{2}^{*}\right)-\left[1+n\left(\mathcal{E}_{1}^{*}\right)\right]\left[1+n\left(\mathcal{E}_{2}^{*}\right)\right]}{k_{0}+\mathcal{E}_{1}^{*}+\mathcal{E}_{2}^{*}} .
$$

The one-loop self-energy is the sum of Eqs. (4.5)-(4.8) and Eq. (4.9) displayed below. Appendix D computes the same quantity using the Minkowski propagators and obtains exactly the same answer.

Unphysical Cuts: There are some additional contributions to Eq. (4.3). If both $\Lambda_{1}$ and $\Lambda_{2}$ are positive integer multiples of $-i 2 \pi T$, then the numerator of Eq. (4.3) vanishes. However if only one of the $\Lambda_{j}$ is a positive integer multiple of $-i 2 \pi T$, the numerator does not vanish. Since Eq. (4.3) is symmetric under interchange of $\vec{k}_{1} \leftrightarrow \vec{k}_{2}$, it is only necessary to consider the case $\Lambda_{1}=-i \omega_{\ell}, \Lambda_{2}=\mathcal{E}_{2}$ or $-\mathcal{E}_{2}^{*}$ and double the result to obtain

$$
\begin{aligned}
g^{2} \int \frac{d \Omega_{12}}{2 E_{2}} & \sum_{\ell=1}^{\infty} \frac{2 T \Gamma_{1} \omega_{\ell}}{\left(\omega_{\ell}^{2}+\mathcal{E}_{1}^{2}\right)\left(\omega_{\ell}^{2}+\mathcal{E}_{1}^{* 2}\right)} \\
& \times\left(\frac{-1}{k_{0}+i \omega_{\ell}-\mathcal{E}_{2}}+\frac{1}{k_{0}+i \omega_{\ell}+\mathcal{E}_{2}^{*}}\right) .
\end{aligned}
$$

These terms have branch cuts in the lower half-plane at $k_{0}$ $=-i \omega_{\ell}+\mathcal{E}_{2}$ and at $k_{0}=-i \omega_{\ell}-\mathcal{E}_{2}^{*}$. The cuts are unphysical in that they are not entirely due to quasiparticle thresholds. The coefficient of this cut is proportional to the damping rate $\Gamma_{1}$ and is in this sense a higher order effect. Section $\mathrm{V}$ will show that Eq. (4.9) is exactly canceled by two-loop effects. For later comparison, it is useful to return to the term in $\Pi^{I}(\tau, \vec{k})$ whose frequency transform produced this cut:

$$
\begin{aligned}
g^{2} \int d \Omega_{12} \sum_{\ell=1}^{\infty} e^{i \omega / \tau} \frac{2 T \Gamma_{1} \omega_{\ell}}{\left(\omega_{\ell}^{2}+\mathcal{E}_{1}^{2}\right)\left(\omega_{\ell}^{2}+\mathcal{E}_{1}^{* 2}\right)} \\
\times\left(\left[1+n\left(\mathcal{E}_{2}\right)\right] \frac{1}{2 E_{2}} e^{-\mathcal{E}_{2} \tau}+n\left(\mathcal{E}_{2}^{*}\right) \frac{1}{2 E_{2}} e^{\mathcal{E}_{2}^{*} \tau}\right) .
\end{aligned}
$$

Advanced Self-Energy: Since quasiparticle propagator satisfies the KMS condition, the integrand of Eq. (4.1) could equally be written $\mathcal{D}\left(\beta-\tau, \vec{k}_{1}\right) \mathcal{D}\left(\beta-\tau, \vec{k}_{2}\right)$. The Fourier transform to $i \omega_{n}$ is then expressed as

$$
\begin{aligned}
\Pi^{I}\left(i \omega_{n}, \vec{k}\right)= & \frac{g^{2}}{2} \int d \Omega_{12} \sum_{\Lambda_{1}, \Lambda_{2}} f\left(\Lambda_{1}\right) f\left(\Lambda_{2}\right) \\
& \times \frac{e^{-\left(\Lambda_{1}+\Lambda_{2}\right) \beta}-1}{i \omega_{n}+\Lambda_{1}+\Lambda_{2}} .
\end{aligned}
$$

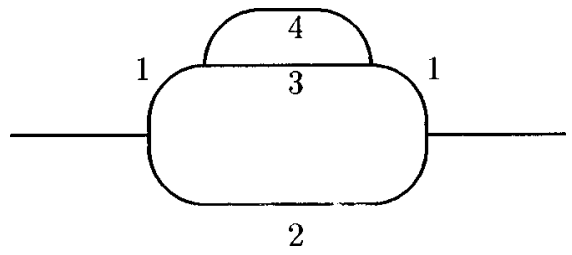

FIG. 2. Two-loop self-energy due to one self-energy insertion.

This is exactly the same self-energy as Eq. (4.3). However in this form, it is easily extended from $i \omega_{n}$ to a function of complex $k_{0}$ that is analytic for $\operatorname{Im} k_{0}<0$. This extension gives the advanced self-energy

$$
\begin{aligned}
\Pi_{A}^{I}\left(k_{0}, \vec{k}\right)= & \frac{g^{2}}{2} \int d \Omega_{12} \sum_{\Lambda_{1}, \Lambda_{2}} f\left(\Lambda_{1}\right) f\left(\Lambda_{2}\right) \\
& \times \frac{e^{-\left(\Lambda_{1}+\Lambda_{2}\right) \beta}-1}{k_{0}+\Lambda_{1}+\Lambda_{2}} .
\end{aligned}
$$

It satisfies $\Pi_{A}(k)=\Pi_{R}(-k)$ as required and has all its branch points in the upper half of the complex $k_{0}$ plane.

Mixed Representations: Because of the KMS condition, one can also represent the self-energy using a mixed form $\mathcal{D}\left(\tau, \vec{k}_{1}\right) \mathcal{D}\left(\beta-\tau, \vec{k}_{2}\right)$. This leads to

$$
\begin{aligned}
\Pi^{I}\left(i \omega_{n}, \vec{k}\right)= & \frac{g^{2}}{2} \int d \Omega_{12} \sum_{\Lambda_{1}, \Lambda_{2}} f\left(\Lambda_{1}\right)\left(\Lambda_{2}\right) \\
& \times \frac{e^{-\Lambda_{2} \beta}-e^{-\Lambda_{1} \beta}}{i \omega_{n}-\Lambda_{1}+\Lambda_{2}} .
\end{aligned}
$$

Although this is the same self-energy, this representation cannot be easily extended to either the retarded or the advanced form of the self-energy. In Sec. V B it will be necessary to use the KMS identity in a similar way to manipulate the two-loop self-energy into a form whose Fourier transform will be analytic in the lower half-plane.

\section{TWO-LOOP SELF-ENERGY}

The simplicity of the one-loop calculation makes it likely that the two-loop contributions can be computed by the same method. The contributions of Figs. 2 and 3 will be denoted by $\Pi_{A}^{I I}$ and $\Pi_{B}^{I I}$, respectively.

\section{A. Self-energy insertion on quasiparticle propagator}

The value of the diagram shown in Fig. 2 is

$$
\Pi_{A}^{I I}(\tau, \vec{k})=-g^{2} \int d \Omega_{12} \mathcal{D}^{I}\left(\tau, \vec{k}_{1}\right) \mathcal{D}_{2}\left(\tau, \vec{k}_{2}\right),
$$

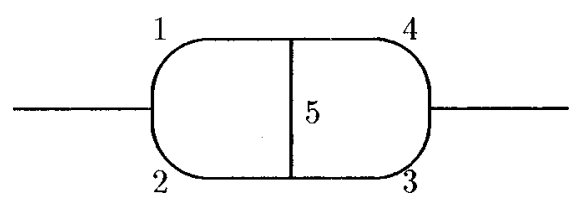

FIG. 3. Two-loop self-energy due to vertex correction. 
where $\mathcal{D}^{I}$ is the one-loop corrected propagator:

$$
\begin{aligned}
\mathcal{D}^{I}(\tau, \vec{k})= & \int_{0}^{\beta} d \tau^{\prime} d \tau^{\prime \prime} \mathcal{D}\left(\tau-\tau^{\prime \prime}, \vec{k}\right) \\
& \times \Pi_{q p}\left(\tau^{\prime \prime}-\tau^{\prime}, \vec{k}\right) \mathcal{D}\left(\tau^{\prime}, \vec{k}\right) .
\end{aligned}
$$

This is not the most convenient way to compute $\mathcal{D}^{I}$. It is easier to employ the method discussed after Eq. (3.10). This requires the Minkowski-space time-ordered propagator, now with one insertion of the retarded and advanced selfenergies:

$$
D_{11}^{I}(k)=\frac{\left[1+n\left(k_{0}\right)\right] \Pi_{R q p}^{I}(k)}{\left(k_{0}-\mathcal{E}\right)^{2}\left(k_{0}+\mathcal{E}^{*}\right)^{2}}-\frac{n\left(k_{0}\right) \Pi_{A q p}^{I}(k)}{\left(k_{0}+\mathcal{E}\right)^{2}\left(k_{0}-\mathcal{E}^{*}\right)^{2}} .
$$

To Fourier transform to real, positive time $t$ requires closing the $k_{0}$ contour in the lower-half of the complex $k_{0}$ plane. The singularities in $k_{0}$ in the lower-half plane are as follows: (1) a simple pole at $k_{0}=\mathcal{E},(2)$ a simple pole at $k_{0}=-\mathcal{E}^{*}$, (3) simple poles in $n\left(k_{0}\right)$ at $k_{0}=-i \omega_{\ell}$, and (4) branch cuts in $\Pi_{R q p}(k)$. Consequently, the Fourier transform is

$$
\begin{aligned}
i D_{11}^{I}(t, \vec{k})= & {[1+n(\mathcal{E})] \frac{B}{2 E} e^{-i \mathcal{E} t}+n\left(\mathcal{E}^{*}\right) \frac{B^{*}}{2 E} e^{i \mathcal{E}^{*} t} } \\
& +T \sum_{\ell=1}^{\infty}\left(\frac{\Pi_{R q p}^{I}(k) e^{-i k_{0} t}}{\left(k_{0}-\mathcal{E}\right)^{2}\left(k_{0}+\mathcal{E}^{*}\right)^{2}}\right)_{k_{0}=-i \omega_{\ell}} \\
& -T \sum_{\ell=1}^{\infty}\left(\frac{\Pi_{A q p}^{I}(k) e^{-i k_{0} t}}{\left(k_{0}+\mathcal{E}\right)^{2}\left(k_{0}-\mathcal{E}^{*}\right)^{2}}\right)_{k_{0}=-i \omega_{\ell}} \\
& +i \int_{\operatorname{Im} k_{0}<0}^{\operatorname{cuts}} \frac{d k_{0}}{2 \pi} D_{11}^{I}\left(k_{0}\right) e^{-i k_{0} t} .
\end{aligned}
$$

The self-energies $\Pi_{R q p}$ and $\Pi_{A q p}$ can be expressed in terms of $\Pi_{R}$ and $\Pi_{A}$ using the definitions (2.7) and (2.14). Evaluating the propagator at the Euclidean time $t=-i \tau$ gives

$$
\begin{aligned}
\mathcal{D}^{I}(\tau, \vec{k})= & {[1+n(\mathcal{E})] \frac{B}{2 E} e^{-\mathcal{E} \tau}+n\left(\mathcal{E}^{*}\right) \frac{B^{*}}{2 E} e^{\mathcal{E}^{*} \tau} } \\
& +T \sum_{\ell=1}^{\infty} e^{i \omega_{\ell} \tau}\left(\frac{2 \Gamma \omega_{\ell}}{\left(\omega_{\ell}^{2}+\mathcal{E}^{2}\right)\left(\omega_{\ell}^{2}+\mathcal{E}^{* 2}\right)}\right. \\
& +\frac{\left.4 \Gamma \omega \ell\left(\omega_{\ell}^{2}+\mathcal{E} \mathcal{E}^{*}\right)\left(\vec{k}^{2}+m^{2}-\mathcal{E E}^{*}\right)+\Gamma^{2} \omega_{\ell}^{2}\right]}{\left(\omega_{\ell}^{2}+\mathcal{E}^{2}\right)^{2}\left(\omega_{\ell}^{2}+\mathcal{E}^{* 2}\right)^{2}} \\
& \left.+\frac{\Pi_{R}^{I}\left(-i \omega_{\ell}\right)}{\left(\omega_{\ell}^{2}+\mathcal{E} \mathcal{E}^{*}-\Gamma \omega_{\ell}\right)^{2}}-\frac{\Pi_{R}^{I}\left(i \omega_{\ell}\right)}{\left(\omega_{\ell}^{2}+\mathcal{E}^{*}+\Gamma \omega_{\ell}\right)^{2}}\right) \\
& +i \int_{\operatorname{Im} k_{0}<0}^{\operatorname{cuts}} \frac{d k_{0}}{2 \pi} D_{11}^{I}\left(k_{0}\right) e^{-k_{0} \tau} .
\end{aligned}
$$

One way of proceeding is to add this correction to the free quasiparticle propagator (3.4). In the sum $\mathcal{D}+\mathcal{D}^{I}$ the coefficients of the quasiparticle terms are modified to $1+B$ and $1+B^{*}$ and the term proportional to $\Gamma$ in Eq. (3.4) cancels in the sum. It was this term that produced the unphysical cuts in the one-loop self-energy. The cancellation in $\mathcal{D}+\mathcal{D}^{I}$ guarantees that unphysical one-loop cuts will be canceled in twoloop order. The following discussion shows these features explicitly as well as the three-quasiparticle cuts that arise.

Wave Function Correction to the Two Quasiparticle Cut: To compute the self-energy requires substituting Eq. (5.2) into Eq. (5.1). The contribution of the first line of Eq. (5.2) to $\Pi_{A}^{I I}(\tau, \vec{k})$ is

$$
\begin{aligned}
& -g^{2} \int d \Omega_{12}\left(\left[1+n\left(\mathcal{E}_{1}\right)\right] \frac{B_{1}}{2 E_{1}} e^{-\mathcal{E}_{1} \tau}+n\left(\mathcal{E}_{1}^{*}\right) \frac{B_{1}^{*}}{2 E_{1}} e^{\mathcal{E}_{1}^{*} \tau}\right) \\
& \times\left(\left[1+n\left(\mathcal{E}_{2}\right)\right] \frac{1}{2 E_{2}} e^{-\mathcal{E}_{2} \tau}+n\left(\mathcal{E}_{2}^{*}\right) \frac{1}{2 E_{2}} e^{\mathcal{E}_{2}^{*} \tau}\right) .
\end{aligned}
$$

This may be symmetrized so that $B_{1}$ and $B_{2}$ appear equally. When added to Eq. (4.2), it merely introduces the wave function correction $1-B_{j} \approx Z_{j}$.

Cancellation of Unphysical Cuts: The $\tau$ dependence in Eq. (4.10) produced the unphysical cuts in Eq. (4.9). When the second line of Eq. (5.2) is substituted into Eq. (5.1), it gives

$$
\begin{aligned}
-g^{2} \int & d \Omega_{12} \sum_{\ell=1}^{\infty} e^{i \omega_{\ell} \tau} \frac{2 T \Gamma_{1} \omega_{\ell}}{\left(\omega_{\ell}^{2}+\mathcal{E}_{1}^{2}\right)\left(\omega_{\ell}^{2}+\mathcal{E}_{1}^{* 2}\right)} \\
& \times\left(\left[1+n\left(\mathcal{E}_{2}\right)\right] \frac{1}{2 E_{2}} e^{-\mathcal{E}_{2} \tau}+n\left(\mathcal{E}_{2}^{*}\right) \frac{1}{2 E_{2}} e^{\mathcal{E}_{2}^{*} \tau}\right) .
\end{aligned}
$$

This exactly cancels Eq. (4.10) so that the one-loop unphysical cuts are removed. Obviously, the third and fourth lines of Eq. (5.2) will produce new unphysical cuts in the two-loop self-energy. These will be canceled by higher loop effects.

Cut for Three Quasiparticles: The last term in Eq. (5.2) requires integrating in $k_{0}$ around the branch cuts in the oneloop self energy:

$$
i \int_{\operatorname{Im} k_{0}<0}^{\text {cuts }} \frac{d k_{0}}{2 \pi} \frac{\Pi_{R}^{I}(k)\left[1+n\left(k_{0}\right)\right]}{\left(k_{0}-\mathcal{E}_{1}\right)^{2}\left(k_{0}+\mathcal{E}_{1}^{*}\right)^{2}} e^{-k_{0} \tau}
$$

It is convenient to use the representation (4.4), but to change the internal momentum variables to $k_{3}$ and $k_{4}$ in correspondence with Fig. 2:

$$
\begin{aligned}
\Pi_{R}^{I}\left(k_{0}\right)= & \frac{g^{2}}{2} \int d \Omega_{34} \sum_{\Lambda_{3}, \Lambda_{4}} f\left(\Lambda_{3}\right) f\left(\Lambda_{4}\right) \\
& \times \frac{1-e^{-\left(\Lambda_{3}+\Lambda_{4}\right) \beta}}{k_{0}-\Lambda_{3}-\Lambda_{4}} .
\end{aligned}
$$

The denominator $k_{0}-\Lambda_{3}-\Lambda_{4}$ produces the branch cut in $k_{0}$. The integration around the cut is performed by interchanging the order of integration to get

$$
\frac{g^{2}}{2} \int d \Omega_{34} \sum_{\Lambda_{3}, \Lambda_{4}} \frac{f\left(\Lambda_{3}\right) f\left(\Lambda_{4}\right) e^{-\left(\Lambda_{3}+\Lambda_{4}\right) \tau}}{\left(\Lambda_{3}+\Lambda_{4}-\mathcal{E}_{1}\right)^{2}\left(\Lambda_{3}+\Lambda_{4}+\mathcal{E}_{1}^{*}\right)^{2}} .
$$


This is the explicit evaluation of $\mathcal{D}_{\text {cut }}^{I}(\tau, \vec{k})$, i.e., the last line of Eq. (5.2). When substituted into Eq. (5.1), the contribution to $\Pi_{A}^{I I}(\tau, \vec{k})$ is

$$
-\frac{g^{4}}{2} \int d \Omega_{12} d \Omega_{34} \sum_{\{\Lambda\}} \frac{f\left(\Lambda_{2}\right) f\left(\Lambda_{3}\right) f\left(\Lambda_{4}\right) e^{-\left(\Lambda_{2}+\Lambda_{3}+\Lambda_{4}\right) \tau}}{\left(\Lambda_{3}+\Lambda_{4}-\mathcal{E}_{1}\right)^{2}\left(\Lambda_{3}+\Lambda_{4}+\mathcal{E}_{1}^{*}\right)^{2}} .
$$

This is easily transformed to get $\Pi_{A}^{I I}\left(i \omega_{n}, \vec{k}\right)$. The extension from $i \omega_{n}$ to complex $k_{0}$ analytic in the upper half-plane is

$$
\begin{aligned}
\Pi_{A}^{I I}\left(k_{0}, \vec{k}\right)= & -\frac{g^{4}}{2} \int d \Omega_{12} d \Omega_{34} \sum_{\{\Lambda\}} \frac{f\left(\Lambda_{2}\right) f\left(\Lambda_{3}\right) f\left(\Lambda_{4}\right)}{k_{0}-\Lambda_{2}-\Lambda_{3}-\Lambda_{4}} \\
& \times \frac{e^{-\left(\Lambda_{2}+\Lambda_{3}+\Lambda_{4}\right) \beta}-1}{\left(\Lambda_{3}+\Lambda_{4}-\mathcal{E}_{1}\right)^{2}\left(\Lambda_{3}+\Lambda_{4}+\mathcal{E}_{1}^{*}\right)^{2}} .
\end{aligned}
$$

This contains the cuts for three quasiparticles at $k_{0}=\Lambda_{2}$ $+\Lambda_{3}+\Lambda_{4}$. The unphysical values of $\Lambda$ will be canceled by higher loops. This completes the analysis of Fig. 2.

\section{B. Vertex correction}

Figure 3 shows the two-loop diagram containing a vertex correction. Two of the loop momenta are independent. For definiteness, the independent momenta are taken as $\vec{k}_{1}$ and $\vec{k}_{3}$ and $d \Omega \equiv d^{3} k_{1} d^{3} k_{3} /(2 \pi)^{6}$. The remaining $\vec{k}_{2}, \vec{k}_{4}, \vec{k}_{5}$ are linear combinations of the these two and the external $\vec{k}$. The self-energy contribution is

$$
\begin{aligned}
\Pi_{B}^{I I}(\tau, \vec{k})= & \frac{g^{4}}{4} \int d \Omega \int_{0}^{\beta} d \tau^{\prime} \int_{0}^{\beta} d \tau^{\prime \prime} \mathcal{D}_{1}\left(\tau^{\prime}\right) \mathcal{D}_{2}\left(\tau^{\prime \prime}\right) \\
& \times \mathcal{D}_{3}\left(\tau^{\prime \prime}-\tau\right) \mathcal{D}_{4}\left(\tau^{\prime}-\tau\right) \mathcal{D}_{5}\left(\tau^{\prime \prime}-\tau^{\prime}\right)
\end{aligned}
$$

The three times $\tau, \tau^{\prime}$, and $\tau^{\prime \prime}$ lie in the interval $[0, \beta]$ and may be ordered in six different ways as follows:

$$
\begin{array}{cc}
B 1: \tau^{\prime}<\tau^{\prime \prime}<\tau & B 2: \tau^{\prime \prime}<\tau^{\prime}<\tau \\
B 3: \tau<\tau^{\prime}<\tau^{\prime \prime} & B 4: \tau<\tau^{\prime \prime}<\tau^{\prime} \\
B 5: \tau^{\prime}<\tau<\tau^{\prime \prime} & B 6: \tau^{\prime \prime}<\tau<\tau^{\prime}
\end{array}
$$

The left and right columns differ by an interchange of $\tau^{\prime}$ and $\tau^{\prime \prime}$. Because of the structure of the integral, this is the same as interchanging $\Lambda_{1} \leftrightarrow \Lambda_{2}$ and $\Lambda_{3} \leftrightarrow \Lambda_{4}$. Thus, only B1, B3, and $\mathrm{B} 5$ need to be computed. With the representation (3.6) for the quasiparticle propagators, the integration over B1 gives

$$
\begin{aligned}
\Pi_{B 1}^{I I}(\tau, \vec{k})= & \frac{g^{4}}{4} \int d \Omega \sum_{\{\Lambda\}} \prod_{j=1}^{5} f\left(\Lambda_{j}\right) \\
& \times\left(\frac{e^{-\left(\Lambda_{1}+\Lambda_{2}\right) \tau}}{\left(\Lambda_{1}-\Lambda_{4}-\Lambda_{5}\right)\left(\Lambda_{1}+\Lambda_{2}-\Lambda_{3}-\Lambda_{4}\right)}\right. \\
& +\frac{e^{-\left(\Lambda_{3}+\Lambda_{4}\right) \tau}}{\left(\Lambda_{2}-\Lambda_{3}+\Lambda_{5}\right)\left(\Lambda_{1}+\Lambda_{2}-\Lambda_{3}-\Lambda_{4}\right)} \\
& \left.+\frac{e^{-\left(\Lambda_{2}+\Lambda_{4}+\Lambda_{5}\right) \tau}}{\left(\Lambda_{1}-\Lambda_{4}-\Lambda_{5}\right)\left(-\Lambda_{2}+\Lambda_{3}-\Lambda_{5}\right)}\right) .
\end{aligned}
$$

The $\tau$ dependence of these three terms will easily lead to two-particle cuts at $k_{0}=\Lambda_{1}+\Lambda_{2}, k_{0}=\Lambda_{3}+\Lambda_{4}$, and a threeparticle cut at $k_{0}=\Lambda_{2}+\Lambda_{4}+\Lambda_{5}$. The next integration, B2, gives the same answer as Eq. (5.8), but with the interchanges $\Lambda_{1} \leftrightarrow \Lambda_{2}$ and $\Lambda_{3} \leftrightarrow \Lambda_{4}$.

Integration B3 can best be done by using the KuboMartin-Schwinger (KMS) condition to rewrite it as

$$
\begin{aligned}
\Pi_{B 3}^{I I}(\tau, \vec{k})= & \frac{g^{4}}{4} \int d \Omega \int_{\tau}^{\beta} d \tau^{\prime} \int_{\tau^{\prime}}^{\beta} d \tau^{\prime \prime} \mathcal{D}_{1}\left(\tau^{\prime}\right) \mathcal{D}_{2}\left(\tau^{\prime \prime}\right) \\
& \mathcal{D}_{3}\left(\beta+\tau-\tau^{\prime \prime}\right) \mathcal{D}_{4}\left(\beta+\tau-\tau^{\prime}\right) \mathcal{D}_{5}\left(\beta+\tau^{\prime}-\tau^{\prime \prime}\right)
\end{aligned}
$$

The time argument for each of the quasiparticle propagators is positive. For example, for $\mathcal{D}_{3}$ the time dependence is $\exp \left[-\Lambda_{3}\left(\beta+\tau-\tau^{\prime \prime}\right)\right]$. The integrand written in this form leads to the most convenient form for the final answer with $\Pi(\tau)$ a product of terms of the form $\exp (-\Lambda \tau)$ as desired. Direct integration gives

$$
\begin{aligned}
\Pi_{B 3}^{I I}(\tau, \vec{k})= & \frac{g^{4}}{4} \int d \Omega \sum_{\{\Lambda\}} \prod_{j=1}^{5} f\left(\Lambda_{j}\right) \\
& \times\left(\frac{e^{-\left(\Lambda_{1}+\Lambda_{2}\right) \tau} e^{-\left(\Lambda_{3}+\Lambda_{4}+\Lambda_{5}\right) \beta}}{\left(\Lambda_{2}-\Lambda_{3}-\Lambda_{5}\right)\left(\Lambda_{1}+\Lambda_{2}-\Lambda_{3}-\Lambda_{4}\right)}\right. \\
& +\frac{e^{-\left(\Lambda_{3}+\Lambda_{4}\right) \tau} e^{-\left(\Lambda_{1}+\Lambda_{2}+\Lambda_{5}\right) \beta}}{\left(\Lambda_{1}-\Lambda_{4}+\Lambda_{5}\right)\left(\Lambda_{1}+\Lambda_{2}-\Lambda_{3}-\Lambda_{4}\right)} \\
& \left.+\frac{e^{-\left(\Lambda_{1}+\Lambda_{3}+\Lambda_{5}\right) \tau} e^{-\left(\Lambda_{2}+\Lambda_{4}\right) \beta}}{\left(\Lambda_{1}-\Lambda_{4}-\Lambda_{5}\right)\left(-\Lambda_{2}+\Lambda_{3}+\Lambda_{5}\right)}\right) .
\end{aligned}
$$

The tau dependence of these terms will again produce two particle cuts at $k_{0}=\Lambda_{1}+\Lambda_{2}, k_{0}=\Lambda_{3}+\Lambda_{4}$, but a different three-particle cut at $k_{0}=\Lambda_{1}+\Lambda_{3}+\Lambda_{5}$. Integration B4 requires interchanging $\Lambda_{1} \leftrightarrow \Lambda_{2}$ and $\Lambda_{3} \leftrightarrow \Lambda_{4}$.

The contribution of B5 is more difficult. First use the KMS condition to write it as

$$
\begin{aligned}
\Pi_{B 5}^{I I}(\tau, \vec{k})= & \frac{g^{4}}{4} \int d \Omega \int_{0}^{\tau} d \tau^{\prime} \int_{\tau}^{\beta} d \tau^{\prime \prime} \mathcal{D}_{1}\left(\tau^{\prime}\right) \mathcal{D}_{2}\left(\tau^{\prime \prime}\right) \\
& \times \mathcal{D}_{3}\left(\beta+\tau-\tau^{\prime \prime}\right) \mathcal{D}_{4}\left(\tau-\tau^{\prime}\right) \mathcal{D}_{5}\left(\beta+\tau^{\prime}-\tau^{\prime \prime}\right)
\end{aligned}
$$

The integration gives 


$$
\begin{aligned}
\Pi_{B 5}^{I I}(\tau, \vec{k})= & \frac{g^{4}}{4} \int d \Omega \sum_{\{\Lambda\}} \prod_{j=1}^{5} f\left(\Lambda_{j}\right) C \\
& \times\left(e^{-\left(\Lambda_{1}+\Lambda_{2}\right) \tau} e^{-\left(\Lambda_{3}+\Lambda_{5}\right) \beta}\right. \\
& +e^{-\left(\Lambda_{3}+\Lambda_{4}\right) \tau} e^{-\Lambda_{2} \beta}-e^{-\left(\Lambda_{1}+\Lambda_{3}+\Lambda_{5}\right) \tau} e^{-\Lambda_{2} \beta} \\
& \left.-e^{\left(-\Lambda_{2}-\Lambda_{4}+\Lambda_{5}\right) \tau} e^{-\left(\Lambda_{3}+\Lambda_{5}\right) \beta}\right) \\
C \equiv & \frac{1}{\left(\Lambda_{1}-\Lambda_{4}+\Lambda_{5}\right)\left(-\Lambda_{2}+\Lambda_{3}+\Lambda_{5}\right)}
\end{aligned}
$$

The last term contains tau dependence $\exp \left(+\Lambda_{5} \tau\right)$ which, when Fourier transformed, is difficult to extend analytically in the upper half-plane. It is useful to isolate all the $\Lambda_{5}$ dependence of this term by defining

$$
Q \equiv \frac{e^{-\Lambda_{5}(\beta-\tau)}}{\left(\Lambda_{1}-\Lambda_{4}+\Lambda_{5}\right)\left(-\Lambda_{2}+\Lambda_{3}+\Lambda_{5}\right)} .
$$

The generalized KMS relation (C9) proven in Appendix C shows that

$$
\begin{aligned}
\sum_{\Lambda_{5}} f\left(\Lambda_{5}\right) Q= & \sum_{\Lambda_{5}}\left(\frac{f\left(\Lambda_{5}\right) e^{-\Lambda_{5} \tau}}{\left(\Lambda_{1}-\Lambda_{4}-\Lambda_{5}\right)\left(-\Lambda_{2}+\Lambda_{3}-\Lambda_{5}\right)}\right. \\
& \left.+\frac{e^{\left(-\Lambda_{1}+\Lambda_{4}\right) \tau} F_{5}\left(-\Lambda_{1}+\Lambda_{4}\right)-e^{\left(-\Lambda_{2}+\Lambda_{3}\right)(\beta-\tau)} F_{5}\left(-\Lambda_{2}+\Lambda_{3}\right)}{\Lambda_{1}+\Lambda_{2}-\Lambda_{3}-\Lambda_{4}}\right)
\end{aligned}
$$

where $F$ is the function defined in Eq. (C1). When this is substituted into Eq. (5.10), the result is

$$
\begin{aligned}
\Pi_{B 5}^{I I}(\tau, \vec{k})= & \frac{g^{4}}{4} \int d \Omega \sum_{\{\Lambda\}} \prod_{j=1}^{4} f\left(\Lambda_{j}\right)\left(f\left(\Lambda_{5}\right) \frac{e^{-\left(\Lambda_{1}+\Lambda_{2}\right) \tau} e^{-\left(\Lambda_{3}+\Lambda_{5}\right) \beta}+e^{-\left(\Lambda_{3}+\Lambda_{4}\right) \tau} e^{-\Lambda_{2} \beta}-e^{-\left(\Lambda_{1}+\Lambda_{3}+\Lambda_{5}\right) \tau} e^{-\Lambda_{2} \beta}}{\left(\Lambda_{1}-\Lambda_{4}+\Lambda_{5}\right)\left(-\Lambda_{2}+\Lambda_{3}+\Lambda_{5}\right)}\right. \\
& \left.\times f\left(\Lambda_{5}\right) \frac{e^{-\left(\Lambda_{2}+\Lambda_{4}+\Lambda_{5}\right) \tau} e^{-\Lambda_{3} \beta}}{\left(\Lambda_{1}-\Lambda_{4}-\Lambda_{5}\right)\left(\Lambda_{2}-\Lambda_{3}+\Lambda_{5}\right)}-\frac{e^{-\left(\Lambda_{1}+\Lambda_{2}\right) \tau} e^{-\Lambda_{3} \beta} F_{5}\left(-\Lambda_{1}+\Lambda_{4}\right)-e^{-\left(\Lambda_{3}+\Lambda_{4}\right) \tau} e^{-\Lambda_{2} \beta} F_{5}\left(-\Lambda_{2}+\Lambda_{3}\right)}{\Lambda_{1}+\Lambda_{2}-\Lambda_{3}-\Lambda_{4}}\right) .
\end{aligned}
$$

The $\tau$ dependence determines the $k_{0}$ dependence. The terms $\exp \left[-\left(\Lambda_{1}+\Lambda_{2}\right) \tau\right]$ and $\exp \left[-\left(\Lambda_{3}+\Lambda_{4}\right) \tau\right]$ produce two particle cuts at $k_{0}=\Lambda_{1}+\Lambda_{2}$ and $k_{0}=\Lambda_{3}+\Lambda_{4}$. The terms $\exp \left[-\left(\Lambda_{1}+\Lambda_{3}+\Lambda_{5}\right) \tau\right]$ and $\exp \left[-\left(\Lambda_{2}+\Lambda_{4}+\Lambda_{5}\right) \tau\right]$ produce three particle cuts at $k_{0}=\Lambda_{1}+\Lambda_{3}+\Lambda_{5}$ and $k_{0}=\Lambda_{2}+\Lambda_{4}$ $+\Lambda_{5}$. Integration B6 requires interchanging $\Lambda_{1} \leftrightarrow \Lambda_{2}$ and $\Lambda_{3} \leftrightarrow \Lambda_{4}$.

\section{CONCLUSION}

The above results follow from the existence of poles in the full retarded propagator $D_{R}^{\prime}\left(k_{0}, \vec{k}_{j}\right)$ at energies $k_{0}=\lambda_{j}$ where

$$
\lambda_{j}=\mathcal{E}\left(\vec{k}_{j}\right) \text { or }-\mathcal{E}^{*}\left(\vec{k}_{j}\right) \operatorname{Im} \lambda<0 .
$$

These poles were shown to produce singularities in retarded self-energy integrands. In the two-quasiparticle channels, there are singularities at $k_{0}=\lambda_{1}+\lambda_{2}$. In the threequasiparticle channels, the singularities are at $k_{0}=\lambda_{1}+\lambda_{2}$ $+\lambda_{3}$. Contributions with $\lambda=\mathcal{E}$ correspond to stimulated emission of quasiparticles weighted by $1+n(\mathcal{E})$; contributions with $\lambda=-\mathcal{E}^{*}$ correspond to absorption of quasiparticles weighted by $n\left(\mathcal{E}^{*}\right)$.

The singularities in the integrands of $\Pi_{R}(k)$ produce branch points when they are trapped at end points of the three-momentum integrations. Without knowing the momentum dependence of $\mathcal{E}(\vec{k})$, it is only possible to analyze this trapping in the equal mass case, i.e., when all the internal lines have the same dispersion relation $\mathcal{E}(\vec{k})$. In that case the pole of the integrand at $k_{0}=\mathcal{E}\left(\vec{k}_{1}\right)+\mathcal{E}\left(\vec{k}_{2}\right)$ produces an end point singularity from $\vec{k}_{1}=\vec{k}_{2}=\vec{k} / 2$. The branch point is thus at $k_{0}=2 \mathcal{E}(\vec{k} / 2)$. For 3 quasiparticles, the branch point is at $k_{0}=3 \mathcal{E}(\vec{k} / 3)$. The poles of the integrand at $k_{0}=\mathcal{E}\left(\vec{k}_{1}\right)$ $-\mathcal{E}\left(\vec{k}_{2}\right) *$ and $k_{0}=-\mathcal{E}\left(\vec{k}_{1}\right) *+\mathcal{E}\left(\vec{k}_{2}\right)$ produce end point singularities from the region $\vec{k}_{1}=\alpha \vec{k}, \vec{k}_{2}=(1-\alpha) \vec{k}$ where $\alpha$ $\rightarrow \pm \infty$. Since all radiative corrections vanish at infinite momentum, the branch points are near the real axis at $k_{0}$ $= \pm|\vec{k}|-i \eta$. These results hold only for equal masses. In general, the branch point locations will depend upon the functions $\mathcal{E}(\vec{k})$.

Cuts in the retarded propagator automatically give those of the advanced propagator because $D_{A}^{\prime}(k)=D_{R}^{\prime}(-k)$. This also determines the four real-time propagators

$$
\begin{aligned}
& D_{11}^{\prime}(k)=\left[1+n\left(k_{0}\right)\right] D_{R}^{\prime}(k)-n\left(k_{0}\right) D_{A}^{\prime}(k) \\
& D_{12}^{\prime}(k)=e^{\sigma k_{0}} n\left(k_{0}\right)\left[D_{R}^{\prime}(k)-D_{A}^{\prime}(k)\right] \\
& D_{21}^{\prime}(k)=e^{(\beta-\sigma) k_{0}} n\left(k_{0}\right)\left[D_{R}^{\prime}(k)-D_{A}^{\prime}(k)\right] \\
& D_{22}^{\prime}(k)=n\left(k_{0}\right) D_{R}^{\prime}(k)-\left[1+n\left(k_{0}\right)\right] D_{A}^{\prime}(k) .
\end{aligned}
$$


Each branch cut of the $D_{a b}^{\prime}$ is completely below the real axis or completely above. There are no branch cuts that cross the real axis. In addition, the $D_{a b}^{\prime}$ have simple poles at $k_{0}$ $= \pm i 2 \pi n T$ from the Bose-Einstein functions. Although the $D_{a b}^{\prime}$ can be written in terms of the thermal Feynman propagators $D_{F / \bar{F}}$, this introduces step functions $\theta\left(k_{0}\right)$ which make the analytic properties of $D_{F / \bar{F}}$ more complicated.

The real-time self-energies are related to the inverse full propagator by

$$
\left[D^{\prime}(k)\right]_{a b}^{-1}=\left(k^{2}-m^{2}\right) \sigma_{a b}^{3}-\Pi_{a b}(k) .
$$

In terms of the retarded and advanced self-energies this implies

$$
\begin{aligned}
& \Pi_{11}(k)=\left[1+n\left(k_{0}\right)\right] \Pi_{R}(k)-n\left(k_{0}\right) \Pi_{A}(k) \\
& \Pi_{12}(k)=e^{\sigma k_{0}} n\left(k_{0}\right)\left[-\Pi_{R}(k)+\Pi_{A}(k)\right] \\
& \Pi_{21}(k)=e^{(\beta-\sigma) k_{0}} n\left(k_{0}\right)\left[-\Pi_{R}(k)+\Pi_{A}(k)\right] \\
& \Pi_{22}(k)=n\left(k_{0}\right) \Pi_{R}(k)-\left[1+n\left(k_{0}\right)\right] \Pi_{A}(k) .
\end{aligned}
$$

Several interesting points require further investigation. The separation of free quasiparticle effects was done by rearranging the propagator. It would be useful to have a operator method for separating the free quasiparticles from the interactions. Work on this is in progress. A related problem is whether the discontinuities can be computed directly without having to compute the entire self-energy as done here. In the perturbative approach, the cutting rules of Kobes and Semenoff [10] accomplish this. However their derivation also requires using the operator structure. The physical significance of the discontinuities requires further investigation. Since the true branch points lie off the real $k_{0}$ axis, it is natural that the discontinuities across the branch cuts are complex. For example, the two-particle discontinuity of Eq. (4.5) is

$$
\begin{aligned}
\operatorname{Disc} \Pi_{R}(k)= & -i \frac{g^{2}}{2} \int \frac{d \Omega_{12}}{2 E_{1} 2 E_{2}} 2 \pi \delta\left(k_{0}-\mathcal{E}_{1}-\mathcal{E}_{2}\right) \\
& \times\left\{\left[1+n\left(\mathcal{E}_{1}\right)\right]\left[1+n\left(\mathcal{E}_{2}\right)\right]-n\left(\mathcal{E}_{1}\right) n\left(\mathcal{E}_{2}\right)\right\}
\end{aligned}
$$

This is very much like what would be expected for the difference between the production rate of two quasiparticles minus their absorption rate, except that the quasiparticle energies $\mathcal{E}$ are complex.

\section{ACKNOWLEDGMENTS}

This work was supported in part by National Science Foundation Grant No. PHY-9630149. It is a pleasure to thank the Institute for Nuclear Theory at the University of Washington and the Department of Energy for partial support during the completion of this work.

\section{APPENDIX A: BREAKDOWN OF PERTURBATION THEORY}

If one applies the Kobes-Semenoff cutting rules [10] to Fig. 2 using free thermal propagators, it has the same breakdown near threshold as the $T=0$ example discussed in Sec. I A. The formula for this particular discontinuity is displayed in Le Bellac [14] and in Gelis [15]. The two-particle discontinuity is

$$
\begin{aligned}
\operatorname{Disc} \Pi_{R}(k)= & \frac{-i g^{2}}{8 \pi^{2}} \int d^{4} p\left[1+n\left(p_{0}\right)+n\left(k_{0}-p_{0}\right)\right] \\
& \times \epsilon\left(p_{0}\right) \delta^{\prime}\left(p^{2}-m^{2}\right) \operatorname{Re} \Pi_{R}\left(p_{0}\right) \\
& \times \epsilon\left(k_{0}-p_{0}\right) \delta\left[(p-k)^{2}-m^{2}\right] .
\end{aligned}
$$

The contribution of $\operatorname{Im} \Pi_{R}$ has been dropped, since it produces a three-particle discontinuity. To display the result, it is useful to let $k=|\vec{k}|$ and $K^{2}=k_{0}^{2}-\vec{k}^{2}$ and $\alpha=(1$ $\left.-4 m^{2} / K^{2}\right)^{1 / 2}$. Direct integration gives

$$
\begin{aligned}
\operatorname{Disc} \Pi_{R}(k)= & \frac{-i g^{2}}{32 \pi \alpha k K^{2}}\left[1+n\left(\frac{k_{0}+\alpha k}{2}\right)+n\left(\frac{k_{0}-\alpha k}{2}\right)\right] \\
& \times\left[\left(k+\alpha k_{0}\right) \operatorname{Re} \Pi_{R}\left(\frac{k_{0}-\alpha k}{2}\right)\right. \\
& \left.+\left(k-\alpha k_{0}\right) \operatorname{Re} \Pi_{R}\left(\frac{k_{0}+\alpha k}{2}\right)\right] \epsilon\left(k_{0}-\alpha k\right)
\end{aligned}
$$

where kinematics requires that either $K^{2}<0$ or $K^{2}>4 m^{2}$. At the perturbative two-particle threshold, $K^{2} \rightarrow 4 \mathrm{~m}^{2}$, so that $\alpha \rightarrow 0$ and

$$
\operatorname{Disc} \Pi_{R}(k) \rightarrow \frac{-i g^{2}}{16 \pi \alpha K^{2}}\left[1+2 n\left(\frac{k_{0}}{2}\right)\right] \operatorname{Re} \Pi_{R}\left(\frac{k_{0}}{2}\right)
$$

The behavior of this discontinuity like $\left(1-4 m^{2} / K^{2}\right)^{-1 / 2}$ produces an infinite correction at the false threshold which signals the breakdown of perturbation theory just as in the zero-temperature example of Sec. I A. One can also check from Eq. (A2) that at the lightcone threshold, $\mathrm{K}^{2} \rightarrow 0^{-}$, the discontinuity does not diverge. In retrospect, this is because the quasiparticle effects do not change the location of the space-like branch cut for equal masses, $-|\vec{k}|<k_{0}<\mid \vec{k}$, as discussed in Sec. VI.

\section{APPENDIX B: REALITY AND KMS CONDITIONS}

It is not obvious that the quasiparticle propagator $\mathcal{D}(\tau, \vec{k})$ displayed in Eq. (3.4) and used throughout the paper satisfies the reality and KMS conditions claimed in Eqs. (3.8) and (3.9). The infinite sum in Eq. (3.4) obscures these properties. One can rewrite that sum in another way using

$$
e^{i \omega_{n}|\tau|}=e^{-i \omega_{n}|\tau|}+2 i \sin \left(\omega_{n}|\tau|\right)
$$


The sum over $\sin \left(\omega_{n} \mid \tau\right)$ can be performed using the identity

$$
\begin{aligned}
T \sum_{n=1}^{\infty} & \sin \left(\omega_{n}|\tau|\right) \frac{-2 i \Gamma \omega_{n}}{\left(\omega_{n}^{2}+\mathcal{E}^{2}\right)\left(\omega_{n}^{2}+\mathcal{E}^{* 2}\right)} \\
= & \frac{1}{4 E}\left\{-[1+n(\mathcal{E})] e^{-\mathcal{E}|\tau|}-n\left(\mathcal{E}^{*}\right) e^{\mathcal{E}^{*}|\tau|}\right. \\
& \left.+\left[1+n\left(\mathcal{E}^{*}\right)\right] e^{-\mathcal{E}^{*}|\tau|}+n(\mathcal{E}) e^{\mathcal{E}|\tau|}\right\} .
\end{aligned}
$$

Using this in Eq. (3.4) gives

$$
\begin{aligned}
\mathcal{D}(\tau, \vec{k})= & \frac{1}{2 E}\left\{\left[1+n\left(\mathcal{E}^{*}\right)\right] e^{-\mathcal{E}^{*}|\tau|}+n(\mathcal{E}) e^{\mathcal{E}|\tau|}\right\} \\
& -T \sum_{n=1}^{\infty} e^{-i \omega_{n}|\tau|} \frac{2 \Gamma \omega_{n}}{\left(\omega_{n}^{2}+\mathcal{E}^{2}\right)\left(\omega_{n}^{2}+\mathcal{E}^{* 2}\right)} .
\end{aligned}
$$

Each term on the right hand side is the complex conjugate of the corresponding term in the original expression (3.4). Hence, $\mathcal{D}(\tau, \vec{k})$ is real.

To prove that the quasiparticle propagator satisfies the KMS condition requires writing the propagator in yet another way. In the original form (3.4) use

$$
e^{i \omega_{n}|\tau|}=\cos \left(\omega_{n}|\tau|\right)+i \sin \left(\omega_{n}|\tau|\right) .
$$

The sum over $\sin \left(\omega_{n} \mid \tau\right)$ can be performed with the identity (B1) to give the result

$$
\begin{aligned}
\mathcal{D}(\tau, \vec{k})= & \frac{1}{4 E}\left\{[1+n(\mathcal{E})] e^{-\mathcal{E}|\tau|}+n\left(\mathcal{E}^{*}\right) e^{\mathcal{E}^{*}|\tau|}\right. \\
& \left.+\left[1+n\left(\mathcal{E}^{*}\right)\right] e^{-\mathcal{E}^{*}|\tau|}+n(\mathcal{E}) e^{\mathcal{E}|\tau|}\right\} \\
& -T \sum_{n=1}^{\infty} \cos \left(\omega_{n}|\tau|\right) \frac{2 \Gamma \omega_{n}}{\left(\omega_{n}^{2}+\mathcal{E}^{2}\right)\left(\omega_{n}^{2}+\mathcal{E}^{* 2}\right)}
\end{aligned}
$$

In this form the KMS condition $\mathcal{D}(\beta-\tau, \vec{k})=\mathcal{D}(\tau, \vec{k})$ is satisfied manifestly.

\section{APPENDIX C: GENERALIZED KMS IDENTITIES}

In Sec. V B it is necessary to use some relations that are generalizations of the KMS identity. To demonstrate these, it is useful to define

$$
F\left(k_{0}\right)=\frac{n\left(k_{0}\right)}{\left(k_{0}-\mathcal{E}\right)\left(k_{0}+\mathcal{E}^{*}\right)}-\frac{n\left(k_{0}\right)}{\left(k_{0}+\mathcal{E}\right)\left(k_{0}-\mathcal{E}^{*}\right)} .
$$

This satisfies

$$
F\left(-k_{0}\right)=e^{\beta k_{0}} F\left(k_{0}\right) .
$$

$F$ has poles in the lower-half of the complex $k_{0}$ plane at $k_{0}$ $=\Lambda$ where $\Lambda \in\left\{\mathcal{E},-\mathcal{E}^{*},-i \omega_{n}\right\}$. At the poles

$$
F\left(k_{0}\right) \rightarrow \frac{e^{-\Lambda \beta} f(\Lambda)}{k_{0}-\Lambda},
$$

where $f(\Lambda)$ are the functions given in Eq. (3.7). It also has poles in the upper half-plane at $k_{0}=-\Lambda$ :

$$
F\left(k_{0}\right) \rightarrow \frac{-f(\Lambda)}{k_{0}+\Lambda} .
$$

KMS Identity: Because $F\left(k_{0}\right)$ vanishes sufficiently rapidly in all directions of the complex plane as $\left|k_{0}\right| \rightarrow \infty$, the contour integral (C3) vanishes when the contour $C$ is a circle of infinite radius:

$$
0=\oint_{C} \frac{d k_{0}}{2 \pi i} F\left(k_{0}\right) e^{k_{0}(\beta-\tau)} \quad(0 \leqslant \tau \leqslant \beta) .
$$

The vanishing of the integral implies that the residues of the lower half-plane poles cancel those of the upper half-plane:

$$
\sum_{\Lambda} f(\Lambda) e^{-\Lambda(\beta-\tau)}=\sum_{\Lambda} f(\Lambda) e^{-\Lambda \tau}
$$

Since the left and right sides of this are the Euclidean propagator (3.6), this just proves the KMS theorem

$$
\mathcal{D}(\beta-\tau, \vec{k})=\mathcal{D}(\tau, \vec{k}) .
$$

Theorem 1: For $C$ a circular contour at infinity and $x$ any complex number inside the contour, the following integral vanishes

$$
0=\oint_{C} \frac{d k_{0}}{2 \pi i} F\left(k_{0}\right) \frac{e^{k_{0}(\beta-\tau)}}{k_{0}-x}(0 \leqslant \tau \leqslant \beta) .
$$

The contribution to the integral of the poles at $k_{0}=\Lambda, k_{0}$ $=-\Lambda$, and $k_{0}=x$ must all cancel. This implies

$$
\sum_{\Lambda} f(\Lambda) \frac{e^{-\Lambda(\beta-\tau)}}{\Lambda+x}=\sum_{\Lambda} f(\Lambda) \frac{e^{-\Lambda \tau}}{-\Lambda+x}-F(x) e^{x(\beta-\tau)} .
$$

This is a generalization of the KMS identity. If the differential operator $(x+d / d \tau)$ is applied to both sides of Eq. (C7), it reduces to Eq. (C4).

Theorem 2: For the same contour as before and $0 \leqslant \tau \leqslant \beta$, the integral (C8) vanishes

$$
0=\oint_{C} \frac{d k_{0}}{2 \pi i} F\left(k_{0}\right) \frac{e^{k_{0}(\beta-\tau)}}{\left(k_{0}-x\right)\left(k_{0}-y\right)} .
$$

Evaluating the integral by Cauchy's theorem gives

$$
\begin{aligned}
\sum_{\Lambda} f(\Lambda) \frac{e^{-\Lambda(\beta-\tau)}}{(\Lambda+x)(\Lambda+y)}= & \sum_{\Lambda} f(\Lambda) \frac{e^{-\Lambda \tau}}{(-\Lambda+x)(-\Lambda+y)} \\
& +\frac{F(x) e^{x(\beta-\tau)}-F(y) e^{y(\beta-\tau)}}{x-y} .
\end{aligned}
$$


Applying $(y+d / d \tau)$, this reproduces Eq. (C7). This identity is used in rewriting Eq. (5.10) in the form (5.11). Obviously, these identities could be generalized to polynomial denominators of any order.

\section{APPENDIX D: ONE-LOOP CALCULATION IN THE REAL-TIME FORMALISM}

Calculations may also be done directly in the real-time formalism. This appendix will compute the one-loop selfenergy in the real-time formalism and show that the answer is the same as obtained rather easily in Sec. IV. In the quasiparticle approximation, the real-time propagators $D_{a b}(k)$ are the linear combinations (6.2) of the approximate retarded and advanced quasiparticle propagators

$$
D_{R}(k)=\frac{1}{\left(k_{0}-\mathcal{E}\right)\left(k_{0}+\mathcal{E}^{*}\right)} \quad D_{A}(k)=\frac{1}{\left(k_{0}+\mathcal{E}\right)\left(k_{0}-\mathcal{E}^{*}\right)} .
$$

The retarded self-energy that implied by Eq. (6.4) is

$$
\left(e^{\beta k_{0}}+1\right) \Pi_{R}(k)=e^{\beta k_{0}} \Pi_{11}(k)-\Pi_{22}(k) .
$$

The one-loop contribution has two propagators with momenta $k_{1}^{\mu}$ and $k_{2}^{\mu}$. Integration will be over $k_{1}$ with the other defined by $k_{2}=k_{1}-k$. The necessary one-loop self-energies are

$$
\begin{aligned}
& \Pi_{11}(k)=\frac{i g^{2}}{2} \int \frac{d^{4} k_{1}}{(2 \pi)^{4}} n\left(k_{1}\right) n\left(k_{2}\right)\left[e^{\beta k_{01}} D_{R}\left(k_{1}\right)-D_{A}\left(k_{1}\right)\right]\left[e^{\beta k_{02}} D_{R}\left(k_{2}\right)-D_{A}\left(k_{2}\right)\right] \\
& \Pi_{22}(k)=\frac{i g^{2}}{2} \int \frac{d^{4} k_{1}}{(2 \pi)^{4}} n\left(k_{1}\right) n\left(k_{2}\right)\left[D_{R}\left(k_{1}\right)-e^{\beta k_{01}} D_{A}\left(k_{1}\right)\right]\left[D_{R}\left(k_{2}\right)-e^{\beta k_{02}} D_{A}\left(k_{2}\right)\right] .
\end{aligned}
$$

When these are substituted into Eq. (D1), the term $D_{A}\left(k_{1}\right) D_{R}\left(k_{2}\right)$ cancels. The remaining three products of the form $D\left(k_{1}\right) D\left(k_{2}\right)$ are multiplied by combinations of exponentials that cancel one of the Bose-Einstein functions $n\left(k_{1}\right)$ or $n\left(k_{2}\right)$. The result is

$$
\begin{aligned}
\left(e^{\beta k_{0}}+1\right) \Pi_{R}(k)= & \frac{i g^{2}}{2} \int \frac{d^{4} k_{1}}{(2 \pi)^{4}} n\left(k_{1}\right) D_{A}\left(k_{2}\right)\left[\left(e^{\beta k_{0}}+1\right) D_{R}\left(k_{1}\right)-\left(e^{\beta k_{0}}+e^{\beta k_{01}}\right) D_{A}\left(k_{1}\right)\right] \\
& +\frac{i g^{2}}{2} \int \frac{d^{4} k_{1}}{(2 \pi)^{4}} n\left(k_{2}\right) D_{R}\left(k_{1}\right)\left[-\left(e^{\beta k_{0}}+1\right) D_{A}\left(k_{2}\right)+\left(e^{\beta k_{01}}+1\right) D_{R}\left(k_{2}\right)\right] .
\end{aligned}
$$

Note that $D_{R}\left(k_{1}\right) D_{A}\left(k_{2}\right)$ appears in both lines. It is convenient to compute the first integral by closing the $k_{01}$ contour below. The poles in the lower half of the $k_{01}$ come from two sources: $D_{R}\left(k_{1}\right)$ has quasiparticle poles at $k_{01}=\mathcal{E}_{1}$ and $k_{01}=-\mathcal{E}_{1}^{*}$ and $n\left(k_{1}\right)$ has poles at $k_{01}=-i \omega \ell$. After the $k_{01}$ integration is performed, there is a common factor $e^{\beta k_{0}}+1$ on the right hand side. The contribution to $\Pi_{R}$ from the first line of Eq. (D3) is

$$
\begin{aligned}
& \frac{g^{2}}{2} \int \frac{d^{3} k_{1}}{(2 \pi)^{3}} \frac{1}{2 E_{1}}\left(\frac{n\left(\mathcal{E}_{1}\right)}{\left(k_{0}-\mathcal{E}_{1}-\mathcal{E}_{2}\right)\left(k_{0}-\mathcal{E}_{1}+\mathcal{E}_{2}^{*}\right)}-\frac{n\left(-\mathcal{E}_{1}^{*}\right)}{\left(k_{0}+\mathcal{E}_{1}^{*}-\mathcal{E}_{2}\right)\left(k_{0}+\mathcal{E}_{1}^{*}+\mathcal{E}_{2}^{*}\right)}\right) \\
& \quad+\frac{g^{2}}{2} \int \frac{d^{3} k_{1}}{(2 \pi)^{3}} T \sum_{\ell=1}^{\infty} \frac{1}{\left(k_{0}+i \omega_{\ell}-\mathcal{E}_{2}\right)\left(k_{0}+i \omega_{\ell}+\mathcal{E}_{2}^{*}\right)} \frac{2 i \omega_{\ell}\left(\mathcal{E}_{1}^{*}-\mathcal{E}_{1}\right)}{\left(\omega_{\ell}^{2}+\mathcal{E}_{1}^{2}\right)\left(\omega_{\ell}^{2}+\mathcal{E}_{1}^{* 2}\right)},
\end{aligned}
$$

where $2 E_{1}=\mathcal{E}_{1}+\mathcal{E}_{1}^{*}$. To compute the integral on the second line of Eq. (D3), it is convenient to close the $k_{01}$ contour above. The poles in the upper-half of the $k_{01}$ plane come from $D_{A}\left(k_{2}\right)$ (recall $k_{02}=k_{01}-k_{0}$ ) at $k_{01}=k_{0}+\mathcal{E}_{2}^{*}$ and $k_{01}=k_{0}-\mathcal{E}_{2}$ and from the Bose-Einstein function $n\left(k_{2}\right)$ at $k_{01}=k_{0}+i \omega_{\ell}$. The second line of Eq. (D3) contributes

$$
\begin{aligned}
& \frac{g^{2}}{2} \int \frac{d^{3} k_{1}}{(2 \pi)^{3}} \frac{1}{2 E_{2}}\left(\frac{n\left(\mathcal{E}_{2}^{*}\right)}{\left(k_{0}+\mathcal{E}_{2}^{*}-\mathcal{E}_{1}\right)\left(k_{0}+\mathcal{E}_{2}^{*}+\mathcal{E}_{1}^{*}\right)}-\frac{n\left(-\mathcal{E}_{2}\right)}{\left(k_{0}-\mathcal{E}_{2}-\mathcal{E}_{1}\right)\left(k_{0}-\mathcal{E}_{2}+\mathcal{E}_{1}^{*}\right)}\right) \\
& +\frac{g^{2}}{2} \int \frac{d^{3} k_{1}}{(2 \pi)^{3}} T \sum_{\ell=1}^{\infty} \frac{1}{\left(k_{0}+i \omega_{\ell}-\mathcal{E}_{1}\right)\left(k_{0}+i \omega_{\ell}+\mathcal{E}_{1}^{*}\right)} \frac{2 i \omega_{\ell}\left(\mathcal{E}_{2}^{*}-\mathcal{E}_{2}\right)}{\left(\omega_{\ell}^{2}+\mathcal{E}_{2}^{2}\right)\left(\omega_{\ell}^{2}+\mathcal{E}_{2}^{* 2}\right)} .
\end{aligned}
$$

The sum of Eqs. (D4) and (D5) gives $\Pi_{R}(k)$ to one-loop order. It agrees completely with the sum of Eqs. (4.5)-(4.9). In this method of calculating, the unphysical branch cuts produced by the denominators containing $k_{0}+i \omega_{\ell}+z$ arise from poles in the Bose-Einstein functions. They are not artifacts of the Euclidean calculation performed in Sec. IV. 
[1] H. A. Weldon, Phys. Rev. D 28, 2007 (1983).

[2] M. A. van Eijck and Ch. G. van Weert, Phys. Lett. B 278, 305 (1992).

[3] M. Jacob and P. V. Landshoff, Phys. Lett. B 281, 114 (1992).

[4] P. Landshoff, Phys. Lett. B 386, 291 (1996).

[5] N. Ashida, H. Kakkagawa, A. Niégawa, and H. Yokota, Phys. Rev. D 45, 2066 (1992).

[6] N. Ashida, H. Kakkagawa, and A. Niégawa, Ann. Phys. (N.Y.) 215, 315 (1992); 230, 161(E) (1994).

[7] A. Niégawa, Phys. Rev. D 57, 1379 (1998).

[8] S. Jeon, Phys. Rev. D 47, 4586 (1993); 52, 3591 (1995).

[9] P. Jizba, Phys. Rev. D 57, 3634 (1998).

[10] R. Kobes and G. Semenoff, Nucl. Phys. B260, 714 (1985); B272, 329 (1986).
[11] R. Kobes, Phys. Rev. D 43, 1269 (1991).

[12] P. F. Bedaque, A. Das, and S. Naik, Phys. Lett. A 12, 2481 (1997).

[13] A. Das, Finite Temperature Field Theory (World Scientific, Singapore, 1997).

[14] M. Le Bellac, Thermal Field Theory (Cambridge University Press, Cambridge, England, 1996).

[15] F. Gelis, Nucl. Phys. B508, 483 (1997).

[16] R. D. Pisarski, Phys. Rev. Lett. 63, 1129 (1989).

[17] E. Braaten and R. D. Pisarski, Phys. Rev. Lett. 64, 1338 (1990); Nucl. Phys. B337, 569 (1990): B339, 310 (1990).

[18] M. Le Bellac and H. Mabilat, Phys. Lett. B 381, 262 (1996); H. Mabilat, Z. Phys. C 75, 155 (1997).

[19] R. D. Pisarski, Nucl. Phys. B309, 476 (1988). 